\title{
Robust Predictive Control of Water Level in an Experimental Pilot Plant with Uncertain Input Delay
}

\author{
Fernanda Quelho Rossi and Roberto Kawakami Harrop Galvão \\ Divisão de Engenharia Eletrônica, Instituto Tecnológico de Aeronáutica, 12228-900 São José dos Campos, SP, Brazil \\ Correspondence should be addressed to Roberto Kawakami Harrop Galvão; kawakami@ita.br
}

Received 2 May 2014; Accepted 19 June 2014; Published 8 July 2014

Academic Editor: Zhan Shu

Copyright ( 2014 F. Q. Rossi and R. K. H. Galvão. This is an open access article distributed under the Creative Commons Attribution License, which permits unrestricted use, distribution, and reproduction in any medium, provided the original work is properly cited.

\begin{abstract}
This paper is concerned with the predictive control of water level in an experimental pilot plant, in the presence of input constraints and uncertain time delays. Robustness is achieved by casting the delay uncertainty into a polytopic form and using a predictive control formulation based on linear matrix inequalities. Integral action is introduced into the controller to ensure offset-free tracking of step changes in the setpoint. Moreover, an integrator resetting procedure based on the concept of regions of guaranteed cost is proposed to improve the resulting transient response. The results show that the introduction of robustness into the predictive control formulation is indeed of value to avoid closed-loop instability in the presence of time delays. In addition, the integrator resetting procedure was found to provide substantial performance improvements in terms of overshoot and settling time.
\end{abstract}

\section{Introduction}

Time delays, often associated with fluid transport processes, measurement procedures, communication lags, and computational processing, may degrade the performance or even cause instability of a control loop [1]. Under such conditions, the design of an appropriate controller can be a challenging task $[2,3]$, especially if the delay is uncertain or subject to unpredictable alterations. In this context, much research effort has been devoted to the development of robust control strategies that guarantee appropriate closed-loop behavior if the delay remains within known bounds [4-6]. Within this scope, robust model-based predictive control (RMPC) may be of particular value in cases where actuator and/or process variable constraints are to be enforced.

The term RMPC refers to a body of receding-horizon optimal control techniques aimed at ensuring stability and constraint satisfaction in the presence of model uncertainties $[7,8]$. Early RMPC formulations involved the online solution of a min-max optimization problem, where the worst case value of the cost function was evaluated over a set of uncertain plants [9-11]. However, the computational cost could be prohibitive for real-time implementation. Within this scope, a significant step forward was made in [12] by casting the optimization problem into a semidefinite programming (SDP) form [13], with model uncertainties and operational constraints incorporated into a set of linear matrix inequalities (LMIs). The control actions can thus be obtained by using efficient numerical methods for convex optimization. Subsequent developments of this RMPC approach have included LMI formulations with less conservatism [14-17], use of output feedback [18-20], extensions to nonlinear systems [21], and handling of asymmetric output constraints [22], among others.

Time-delay systems were briefly mentioned in [12] as a remark concerning possible applications of the proposed RMPC technique. This possibility was formalized in a more recent paper [23], which concerned systems with polytopic uncertainty description and both input and state delays. The proposed formulation was later extended to handle multiple input delays [24], as well as uncertainties in both the model parameters and delay values [25]. A simpler approach can also be adopted if the uncertainty is restricted to the input delay. In this case, the original RMPC formulation of [12] can be employed by using an augmented state vector including current values of the state and past values of 
the control input [26-28]. As can be seen, much research effort has been devoted to the control of time delay systems with RMPC techniques derived from [12]. However, to the best of the authors' knowledge, experimental studies of this RMPC approach for systems with uncertain delays have not been reported, either in laboratory scale or actual industrial practice. Such a gap motivated the present work, which is concerned with the control of water level in an experimental pilot plant in the presence of uncertain input delay.

Tank level control plays an important role in several industrial settings, including, for instance, chemical, pharmaceutical, and paper manufacturing processes [29]. This application has been widely employed in the literature and is often considered a benchmark control problem [30-32]. It is worth noting that the work reported in [32] involved the control of a laboratory tank system by using the RMPC formulation of [12]. However, that work employed a didactic plant of reduced scale and was not concerned with possible time delays. In contrast, the present investigation involves a pilot plant with actual industrial components, with emphasis on potential problems associated with the presence of time delay.

The RMPC approach adopted in this paper follows the formulation proposed in [12]. Uncertainty in the input delay is handled by using an augmented state vector, as in [28]. Moreover, offset-free tracking of step changes in the setpoint is ensured by introducing an integrator associated with the process variable of interest (water level). In order to reduce the overshoot and settling time of the transient response, an integrator resetting procedure is employed [33]. An improved resetting criterion is formulated herein, by using the concept of regions of guaranteed cost [34].

The remainder of this paper is organized as follows. Section 2 describes the LMI-based RMPC formulation adopted in the present work. Section 2.1 presents an overview of the RMPC formulation. Section 2.2 discusses the concept of regions of guaranteed cost, which will be used in the integrator resetting procedure. The handling of uncertain input delays is addressed in Section 2.3. The proposed integrator resetting scheme is presented in Section 3. The case study involving the experimental pilot plant is described in Section 4. Simulation and experimental results are discussed in Section 5. Lastly, concluding remarks are presented in Section 6.

\section{LMI-Based Robust Model Predictive Control}

2.1. Overview of the RMPC Formulation. The RMPC approach under consideration is concerned with discretetime, uncertain state-space models of the form as follows:

$$
x(k+1)=A x(k)+B u(k), \quad(A, B) \in \Omega,
$$

where $x(k) \in \mathbb{R}^{n}$ and $u(k) \in \mathbb{R}^{m}$ are the state and input variables, respectively. It is assumed that matrices $A$ and $B$ belong to an uncertainty polytope $\Omega$ with known vertices $A_{i} \in \mathbb{R}^{n \times n}, B_{i} \in \mathbb{R}^{n \times m}, i=1,2, \ldots, L$; that is,

$$
(A, B)=\sum_{i=1}^{L} \lambda_{i}\left(A_{i}, B_{i}\right),
$$

for some set of scalars $\lambda_{1}, \lambda_{2}, \ldots, \lambda_{L}$ such that

$$
\sum_{i=1}^{L} \lambda_{i}=1, \quad \lambda_{i} \geq 0
$$

Let $J_{\infty}(k)$ denote the following infinite-horizon cost function:

$$
\begin{aligned}
J_{\infty}(k)=\sum_{j=0}^{\infty}[ & x(k+j \mid k)^{T} S x(k+j \mid k) \\
& \left.+u(k+j \mid k)^{T} R u(k+j \mid k)\right],
\end{aligned}
$$

where $S \in \mathbb{R}^{n \times n}$ and $R \in \mathbb{R}^{m \times m}$ are positive-definite weight matrices and $(\bullet \mid k)$ denotes a predicted value, which is computed on the basis of the information available at time $k$. It is assumed that the system state is directly measured, so that $x(k \mid k)=x(k)$.

The optimization problem to be solved at time $k$ can be formulated as

$$
\min _{u(k+j \mid k), j \geq 0} \max _{(A, B) \in \Omega} J_{\infty}(k)
$$

subject to

$$
\left|u_{r}(k+j \mid k)\right| \leq u_{r, \max }, \quad r=1,2, \ldots, m, j \geq 0,
$$

where $u_{r}$ denotes the $r$ th input variable and $u_{r \text {,max }}$ is the corresponding bound on its magnitude.

In the RMPC approach proposed in [12], an upper bound $\gamma$ on the cost $J_{\infty}(k)$ is minimized by solving the following semidefinite programming (SDP) problem:

$$
\min _{\gamma, Q>0, Y, X} \gamma
$$

subject to

$$
\left[\begin{array}{cc}
Q & x(k) \\
* & 1
\end{array}\right] \geq 0
$$

$$
\begin{gathered}
{\left[\begin{array}{cccc}
Q & 0 & 0 & A_{i} Q+B_{i} Y \\
* & \gamma I & 0 & S^{1 / 2} Q \\
* & * & \gamma I & R^{1 / 2} Y \\
* & * & * & Q
\end{array}\right] \geq 0, \quad i=1,2, \ldots, L} \\
{\left[\begin{array}{cc}
X & Y \\
* & Q
\end{array}\right] \geq 0} \\
X_{r r} \leq u_{r, \text { max }}^{2}, \quad r=1,2, \ldots, m,
\end{gathered}
$$

where $X_{r r}$ are the diagonal elements of $X$ and symbol * represents the elements below the main diagonal of a symmetric matrix. In what follows the SDP problem given by (7)-(11) will be termed $\mathbb{P}(x(k))$. 
The resulting control action is given by $u(k)=F x(k)$, with $F=Y Q^{-1}$. By applying this control law in a receding horizon manner, that is, by solving $\mathbb{P}(x(k))$ in order to obtain a new gain matrix $F$ at each sampling time $k$, the closed-loop system can be shown to be robustly asymptotically stable, provided that $\mathbb{P}(x(k))$ is feasible at the initial time $k=0$. A detailed proof of this property can be found in [12].

Henceforth, a subscript $k$ will be included in the solution $\left(\gamma_{k}, Q_{k}, Y_{k}, X_{k}\right)$ of $\mathbb{P}(x(k))$, as well as in the resulting gain matrix $F_{k}=Y_{k} Q_{k}^{-1}$, to indicate the corresponding time index.

Remark 1 (tracking a constant reference). In cases where the target state is an equilibrium point $x_{p \text {,ref }} \neq 0$, associated with a steady-state control $u_{p \text {,ref }}$, the RMPC control law can be implemented by expressing $x(k)$ as a deviation from the equilibrium value; that is,

$$
x(k)=x_{p}(k)-x_{p, \text { ref }},
$$

where $x_{p}(k)$ denotes the actual state of the plant. The actual control $u_{p}(k)$ is obtained by solving problem $\mathbb{P}(x(k))$ and calculating

$$
\begin{gathered}
u(k)=F_{k} x(k) \\
u_{p}(k)=u(k)+u_{p, \text { ref }} .
\end{gathered}
$$

It is worth noting that the constraints on $u_{p}(k)$ must be translated in terms of symmetrical bounds on each component of $u(k)$ for use in the optimization problem $\mathbb{P}(x(k))$.

2.2. Regions of Guaranteed Cost. A region of guaranteed cost $\mathscr{D}^{\bar{\gamma}} \subset \mathbb{R}^{n}$ can be defined as the set of state vectors $x \in \mathbb{R}^{n}$ for which $\mathbb{P}(x)$ is feasible, with optimal cost $\gamma^{*}$ being smaller or equal to a given bound $\bar{\gamma}>0$. This region is convex and symmetric about the origin, as demonstrated in [34].

An extreme point of $\mathscr{D}^{\bar{\gamma}}$ along the direction of a given vector $\xi \in \mathbb{R}^{n}$ can be obtained by solving the following SDP problem [34]:

$$
\min _{\gamma, \mathrm{Q}>0, \beta<0, Y, X} \beta,
$$

subject to

$$
\begin{gathered}
\gamma \leq \bar{\gamma} \\
{\left[\begin{array}{cc}
Q & \beta \xi \\
* & 1
\end{array}\right] \geq 0}
\end{gathered}
$$

and the remaining LMIs (9)-(11) of the original RMPC optimization problem. The resulting extreme point is given by $\beta^{*} \xi$, where $\beta^{*}$ is the minimal value found for $\beta$. Due to the property of symmetry, $-\beta^{*} \xi$ will also be an extreme point. An inner approximation of $\mathscr{D}^{\bar{\gamma}}$ can be generated as the convex hull of extreme points obtained with different $\xi$ vectors.

2.3. Handling of Uncertain Input Delays. In the present work, the time delay is considered to be the main source of uncertainty for the design of the controller. It is assumed that the dynamics of the plant can be described by a model of the form

$$
z(k+1)=\Phi z(k)+\Gamma u(k-\tau),
$$

where $z(k) \in \mathbb{R}^{n_{z}}$ denotes the plant state at time $k, \Phi \in$ $\mathbb{R}^{n_{z} \times n_{z}}, \Gamma \in \mathbb{R}^{n_{z} \times m}$ are known matrices, and $\tau \in \mathbb{N}$ is an uncertain delay, which may take values in the range $[0, \bar{\tau}]$. For simplicity, the formulation presented herein is derived for a single-input system (i.e., $m=1$ ), as in [28]. However, the extension to the multiple-input case does not pose substantial difficulties, involving mostly changes in notation [35].

The RMPC formulation can be applied by adopting the following state vector $x(k) \in \mathbb{R}^{n_{z}+\bar{\tau}}$, which comprises the plant state $z(k)$ and $\bar{\tau}$ past values of control:

$$
x(k) \triangleq\left[\begin{array}{c}
z(k) \\
u(k-1) \\
u(k-2) \\
\vdots \\
u(k-\bar{\tau}+1) \\
u(k-\bar{\tau})
\end{array}\right] .
$$

The dynamics of the plant with uncertain input delay can then be described by a model in the form (1) with

$$
\begin{gathered}
A=\left[\begin{array}{cccccc}
\Phi & \lambda_{1} \Gamma & \lambda_{2} \Gamma & \cdots & \lambda_{\bar{\tau}-1} \Gamma & \lambda_{\bar{\tau}} \Gamma \\
0 & 0 & 0 & \cdots & 0 & 0 \\
0 & 1 & 0 & \cdots & 0 & 0 \\
0 & 0 & 1 & \cdots & 0 & 0 \\
\vdots & \vdots & \vdots & \ddots & \vdots & \vdots \\
0 & 0 & 0 & \cdots & 1 & 0
\end{array}\right] \\
B=\left[\begin{array}{llllll}
\lambda_{0} \Gamma^{T} & 1 & 0 & 0 & \cdots & 0
\end{array}\right]^{T},
\end{gathered}
$$

where the scalars $\lambda_{1}, \lambda_{2}, \ldots, \lambda_{\bar{\tau}}$ are such that

$$
\lambda_{d}= \begin{cases}1, & \text { if } d=\tau \\ 0, & \text { otherwise }\end{cases}
$$

From the definition of $\lambda_{d}$ in (19), it follows that

$$
\begin{gathered}
\lambda_{d} \in\{0,1\}, \quad d=0,1, \ldots, \bar{\tau} \\
\sum_{d=0}^{\bar{\tau}} \lambda_{d}=1 .
\end{gathered}
$$

Now, let $\Lambda=\left(\lambda_{0}, \lambda_{1}, \ldots, \lambda_{\bar{\tau}}\right)$. In view of $(20), \Lambda$ can only assume $\bar{\tau}+1$ values; namely,

$$
\begin{aligned}
& \Lambda_{0}=(1,0, \ldots, 0) \\
& \Lambda_{1}=(0,1, \ldots, 0)
\end{aligned}
$$

$$
\Lambda_{\bar{\tau}}=(0,0, \ldots, 1) .
$$


Therefore, the matrices $(A, B)$ in (18) belong to a polytope $\Omega$ with $L=(\bar{\tau}+1)$ vertices associated with $\Lambda_{0}, \Lambda_{1}, \ldots, \Lambda_{\bar{\tau}}$.

The control algorithm can be summarized in the following form. Given the matrices $\Phi$ and $\Gamma$ of the model (16), as well as the maximum value $\bar{\tau}$ of the time delay, the vertices $\left(A_{i}, B_{i}\right)$ of the uncertainty polytope $\Omega$ are written as

$$
\begin{aligned}
A_{1}=\left[\begin{array}{cccccc}
\Phi & 0 & 0 & \cdots & 0 & 0 \\
0 & 0 & 0 & \cdots & 0 & 0 \\
0 & 1 & 0 & \cdots & 0 & 0 \\
0 & 0 & 1 & \cdots & 0 & 0 \\
\vdots & \vdots & \vdots & \ddots & \vdots & \vdots \\
0 & 0 & 0 & \cdots & 1 & 0
\end{array}\right], & B_{1}=\left[\begin{array}{c}
\Gamma \\
1 \\
0 \\
0 \\
\vdots \\
0
\end{array}\right] \\
A_{2}=\left[\begin{array}{cccccc}
\Phi & \Gamma & 0 & \cdots & 0 & 0 \\
0 & 0 & 0 & \cdots & 0 & 0 \\
0 & 1 & 0 & \cdots & 0 & 0 \\
0 & 0 & 1 & \cdots & 0 & 0 \\
\vdots & \vdots & \vdots & \ddots & \vdots & \vdots \\
0 & 0 & 0 & \cdots & 1 & 0
\end{array}\right], & B_{2}=\left[\begin{array}{c}
0 \\
1 \\
0 \\
0 \\
\vdots \\
0
\end{array}\right] \\
A_{L}= & {\left[\begin{array}{cccccc}
\Phi & 0 & 0 & \cdots & 0 & \Gamma \\
0 & 0 & 0 & \cdots & 0 & 0 \\
0 & 1 & 0 & \cdots & 0 & 0 \\
0 & 0 & 1 & \cdots & 0 & 0 \\
\vdots & \vdots & \vdots & \ddots & \vdots & \vdots \\
0 & 0 & 0 & \cdots & 1 & 0
\end{array}\right], }
\end{aligned}
$$

At the $k$ th sampling time, the state vector $z(k)$ is measured, the augmented state vector $x(k)$ is built as in (17), and the optimization problem (7)-(11) is solved in order to obtain the matrix $F_{k}$. The control $u(k)=F_{k} x(k)$ is then calculated and applied to the plant.

Remark 2. This polytopic description of the input delay uncertainty was adopted in [26-28] as a direct extension of the original RMPC formulation of [12]. This approach can also be employed if the delay is lower-bounded by a known value $\underline{\tau}>0$, in which case the vertices of the uncertainty polytope $\Omega$ corresponding to $\Lambda_{0}, \Lambda_{1}, \ldots, \Lambda_{\tau-1}$ should be removed. If both the time delay and the model parameters were subject to uncertainties, an alternative would consist of using the more elaborate formulation proposed in [25]. However, as will be seen in Section 5, the input delay is indeed the main source of uncertainty in the present work.

\section{Integrator Resetting}

3.1. Integral Control Action. As discussed in Remark 1, the state of the plant can be steered to a desired equilibrium point by expressing the RMPC control law in terms of deviations from the equilibrium values. However, the presence of constant disturbances acting on the plant may lead to steadystate errors. Therefore, if offset-free tracking is required for a particular process variable $y$, some form of integral action should be introduced in the controller $[33,36]$.
For this purpose, assume that $y$ is related to the state $x$ by an output equation of the form

$$
y(k)=C x(k)
$$

where $C \in \mathbb{R}^{1 \times n}$ is a known matrix. If both $y$ and $x$ denote deviations from desired target values, as in Remark 1 , offsetfree tracking will be achieved if $y(k) \rightarrow 0$ as $k \rightarrow \infty$. Integral control action can be introduced by augmenting the plant state with an additional variable $w$ associated with a discrete-time integrator:

$$
w(k+1)=w(k)-y(k) .
$$

If the control loop is stable, $w(k)$ will converge to a constant value as $k \rightarrow \infty$ and thus $y(k)$ will converge to zero [36]. It is worth noting that the use of a minus sign in (24) is an arbitrary convention, as the same reasoning holds if the equation is rewritten as $w(k+1)=w(k)+y(k)$. The minus sign is employed herein for consistency with the notation adopted in [33].

From (1) and (24), the augmented model can be written as

$$
\left[\begin{array}{c}
x(k+1) \\
w(k+1)
\end{array}\right]=\left[\begin{array}{cc}
A & 0 \\
-C & 1
\end{array}\right]\left[\begin{array}{c}
x(k) \\
w(k)
\end{array}\right]+\left[\begin{array}{c}
B \\
0
\end{array}\right] u(k)
$$

where 0 denotes either a scalar zero or a matrix of zeros with appropriate dimensions. The RMPC formulation presented in Section 2 can then be employed by replacing the original state $x(k)$ with the augmented state $\left[x^{T}(k) w(k)\right]^{T}$. The optimal control at each time $k$ will be given by

$$
u(k)=F_{k}\left[\begin{array}{c}
x(k) \\
w(k)
\end{array}\right]
$$

with $F_{k}$ obtained from the solution of $\mathbb{P}\left(\left[x^{T}(k) w(k)\right]^{T}\right)$.

3.2. Integrator Resetting Procedure. The inclusion of an integrator in a control loop may cause undesirable effects in the transient response, such as increase in overshoot and/or settling time. Indeed, a control law such as (26) is aimed at forcing $x(k) \stackrel{k \rightarrow \infty}{\longrightarrow} 0$ and $w(k) \stackrel{k \rightarrow \infty}{\longrightarrow} 0$. The variable $w$ corresponds to the accumulated value of $-y$ (in terms of deviations from the desired target), as defined in (24). Therefore, after the target value is reached (i.e., when $y$ reaches zero), the controller will need to steer the process variable to the opposite side of the target, in order to switch the sign of $y$ and thus move $w$ progressively to zero (possibly in a slow manner). A speed up in this process can be achieved by means of integrator resetting.

A resetting procedure that retains the robust stability properties of the RMPC formulation was introduced in [33]. Such a procedure consisted of replacing the integrator state $w(k)$ with zero when the process variable crossed the setpoint for the first time, provided that the RMPC optimization problem remained feasible.

Herein, an improved scheme of integrator resetting is proposed by using a criterion based on the overall state vector, 
instead of the process variable alone. More specifically, the integrator state $w(k)$ will be replaced with 0 if such a procedure reduces the RMPC cost below a prespecified threshold $\bar{\gamma}$. This condition is tested by checking whether the augmented state $\left[x^{T}(k) 0\right]^{T}$ belongs to the region of guaranteed cost $\mathscr{D}^{\bar{\gamma}}$. In this manner, the subsequent transient performance of the closed-loop system, as evaluated by the cost function, is taken into account. In contrast, it may be argued that the procedure described in [33], which was based on the difference between a single variable and its corresponding setpoint, does not consider the transient response resulting from the resetting intervention.

The proposed procedure can be described as follows.

Step 0 (initialization). Let $\mathscr{D}^{\bar{\gamma}} \subset \mathbb{R}^{n+1}$ be a region of guaranteed cost associated with an upper bound $\bar{\gamma}$ on the cost value.

Let $k=0, w(0)=0$, and FLAG $=0$.

Step 1. Read state $x(k)$.

Step 2 (integrator resetting). If the following conditions are simultaneously satisfied:

$$
\begin{gathered}
{\left[\begin{array}{c}
x(k) \\
0
\end{array}\right] \in \mathscr{D}^{\bar{\gamma}}} \\
F L A G=0
\end{gathered}
$$

then let $w(k)=0$ and FLAG $=1$.

Step 3. Obtain the solution $\left(\gamma_{k}, Q_{k}, Y_{k}, X_{k}\right)$ for the problem $\mathbb{P}\left(\left[x^{T}(k) w(k)\right]^{T}\right)$.

Step 4. Calculate the gain matrix $F_{k}=Y_{k}\left(Q_{k}\right)^{-1}$ and the control input $u(k)=F_{k}\left[x^{T}(k) w(k)\right]^{T}$.

Step 5. Apply $u(k)$ to the plant and let $w(k+1)=w(k)-C x(k)$.

Step 6. Let $k=k+1$; wait until the next sampling time and return to Step 1 .

Remark 3 (resetting conditions). Condition (27), together with the definition of regions of guaranteed cost in Section 2.2 , ensures that problem $\mathbb{P}\left(\left[x^{T}(k) w(k)\right]^{T}\right)$ will be feasible with $w(k)=0$. The FLAG variable indicates whether the integrator has already been reset. Thus, condition (28) ensures that the resetting operation will be carried out only once. This safeguard is of value to retain the stability properties of the RMPC formulation. Indeed, after the integrator is reset, all subsequent control actions will be obtained by following the standard RMPC control law, with no more resetting interventions, and thus the associated robust stability guarantees will apply.

Remark 4 (region of guaranteed cost $\mathscr{D}^{\bar{\gamma}}$ : simplified test). Condition (27) can be replaced by a simpler sufficient test, as described below. Let $x_{1}, x_{2}, \ldots, x_{n}$ denote the components of the state vector $x$. Assume that the following $n$ vectors in
$\mathbb{R}^{n+1}$ are extreme points of $\mathscr{D}^{\bar{\gamma}}$ along the directions of these components:

$$
\left[\begin{array}{c}
p_{1} \\
0 \\
\vdots \\
0 \\
0
\end{array}\right],\left[\begin{array}{c}
0 \\
p_{2} \\
\vdots \\
0 \\
0
\end{array}\right], \ldots,\left[\begin{array}{c}
0 \\
0 \\
\vdots \\
p_{n} \\
0
\end{array}\right]
$$

These extreme points can be obtained by using the search method described in Section 2.2 with vectors $\xi$ of the form $\left[\begin{array}{lllll}1 & 0 & \cdots & 0 & 0\end{array}\right]^{T},\left[\begin{array}{lllll}0 & 1 & \cdots & 0 & 0\end{array}\right]^{T}, \ldots,\left[\begin{array}{lllll}0 & 0 & \cdots & 1 & 0\end{array}\right]^{T}$. Since $\mathscr{D}^{\bar{\gamma}}$ is symmetrical about the origin, the following vectors are also extreme points:

$$
\left[\begin{array}{c}
-p_{1} \\
0 \\
\vdots \\
0 \\
0
\end{array}\right],\left[\begin{array}{c}
0 \\
-p_{2} \\
\vdots \\
0 \\
0
\end{array}\right], \ldots,\left[\begin{array}{c}
0 \\
0 \\
\vdots \\
-p_{n} \\
0
\end{array}\right]
$$

The convexity property ensures that $\mathscr{D}^{\bar{\gamma}}$ contains the convex hull of the extreme points in (29) and (30). Therefore, if

$$
\frac{\left|x_{1}(k)\right|}{\left|p_{1}\right|}+\frac{\left|x_{2}(k)\right|}{\left|p_{2}\right|}+\cdots+\frac{\left|x_{n}(k)\right|}{\left|p_{n}\right|} \leq 1
$$

then $\left[x^{T}(k) 0\right]^{T} \in \mathscr{D}^{\bar{\gamma}}$.

It is worth noting that the integrator resetting procedure described herein can be directly applied to the case of uncertain input delay. It is sufficient to adopt the state vector $x(k)$ as in (17), with the associated polytopic model described in Section 2.3.

\section{Case Study}

The pilot plant employed herein was developed by Yokogawa Electric Corporation for process control experiments involving actual industrial components. As depicted in Figure 1(a), the plant comprises a reservoir tank $\left(T_{R}\right)$, a process tank $\left(T_{P}\right)$, and a heating tank $\left(T_{H}\right)$. Since the present work was only concerned with the control of water level in the process tank $T_{P}$, the connection valves to the heating $\operatorname{tank} T_{H}$ were closed. A simplified scheme of the remaining components of the plant can be seen in Figure 1(b).

The water level in the reservoir tank $T_{R}$ is regulated by means of a simple on/off switch, which was designed to keep this tank at its maximum capacity. A pump connected to a three-phase induction motor (MDP in Figure 1(b)) transfers water from $T_{R}$ to the process tank $T_{P}$. The water leaves $T_{P}$ through a valve at the bottom of the tank. A frequency inverter is used to drive the induction motor and a differential pressure transmitter is employed to measure the water level in $T_{P}$. A stardom programmable logic controller (PLC) is responsible for sending commands to the frequency inverter and reading the level measurements through a fieldbus 


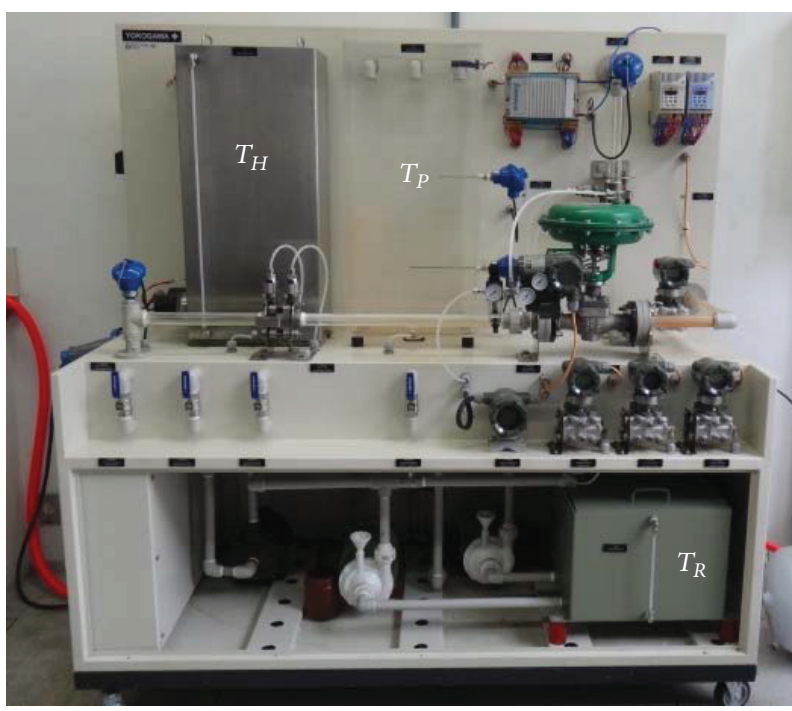

(a)

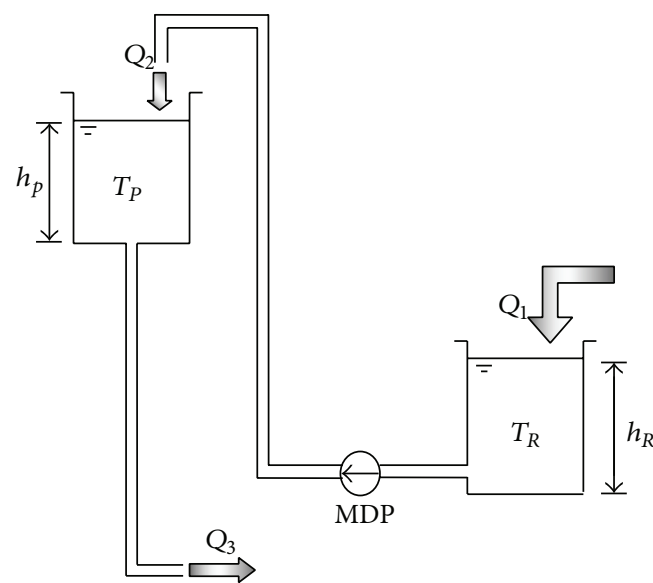

$T_{P}$ : process tank $T_{R}$ : reservoir tank MDP: motor-driven pump $h_{p}$ : water level in $T_{P}$
$h_{R}:$ water level in $T_{R}$
$Q_{1}$ : flow into $T_{R}$
$Q_{2}:$ flow from $T_{R}$ to $T_{P}$
$Q_{3}$ : output flow from $T_{P}$

(b)

FIGURE 1: (a) Experimental pilot plant and (b) schematic representation of the plant section involved in the level control experiments.

network. In addition, the PLC communicates with a personal computer over an Ethernet channel. In the present work, the RMPC control algorithm was implemented in the personal computer by using an OPC (object linking and embedding for process control) protocol to acquire sensor readings (water level in $T_{P}$ ) and send actuator commands (rotation speed of the motor-driven pump) at each sampling time. The LMI toolbox of the Matlab software environment was employed to solve the semidefinite programming problem involved in the RMPC implementation.

4.1. Plant Model. The values of the control input $u_{p}$ (rotation speed) and the process variable of interest $h_{p}$ (water level) were normalized between $0 \%$ and $100 \%$, corresponding to a range of [0-3450] rpm for $u_{p}$ and $[0-70] \mathrm{cm}$ for $h_{p}$. A model of the plant was obtained from open-loop step responses around the equilibrium values $u_{p \text {,ref }}=65 \%$ and $h_{p \text {,ref }}=30 \%$. By using a sampling time $T_{s}=5 \mathrm{~s}$, the resulting model can be written as

$$
h(k+1)=h(k)+0.0865 u(k-1),
$$

where $h$ and $u$ denote deviations from $h_{p}$ and $u_{p}$ from the equilibrium values; that is,

$$
\begin{aligned}
& h(k)=h_{p}(k)-h_{p, \text { ref }} \\
& u(k)=u_{p}(k)-u_{p, \text { ref }},
\end{aligned}
$$

It is worth noting that the model (32) comprises a delay of one sampling period at the plant input. As discussed in [37], this delay is associated with the relation between the actuator command and the water flow to the process tank. Uncertainty was introduced by including an additional delay in the control code running within the Matlab software environment. In practice, such a delay could arise, for example, from failures in communication links of networked control systems [38]. The plant model (32) then becomes

$$
h(k+1)=h(k)+0.0865 u(k-\tau),
$$

where $\tau$ is an uncertain delay with possible values in the range $[1, \bar{\tau}]$. In the present case study, the maximum delay value was taken as $\bar{\tau}=6$.

Equation (35) is in the form (16) with $z(k)=h(k), \Phi=$ 1 , and $\Gamma=0.0865$. Therefore, the delay uncertainty can be handled by using the formulation in Section 2.3 with the state vector $x(k)$ defined as

$$
x(k)=\left[\begin{array}{llllll}
h(k) & u(k-1) & u(k-2) & \cdots & u(k-6)
\end{array}\right]^{T} .
$$

In order to ensure offset-free tracking of a constant reference for the water level, an additional state $w(k)$ was included as in (25), with $C=\left[\begin{array}{llll}1 & 0 & \cdots & 0\end{array}\right]$.

4.2. Controller Design. The control task in this case study consists of increasing the water level from an initial value $h_{p}(0)=12.5 \%$ up to a setpoint $h_{p \text {, ref }}=30 \%$. As mentioned above, the corresponding steady-state rotation of the motordriven pump is approximately $u_{p \text {,ref }}=65 \%$. The precise value of $u_{p}$ required to keep the water level at the desired setpoint depends on the settings of the output valve at the bottom of the process tank. However, possible differences with respect to the adopted value $\left(u_{p, \text { ref }}=65 \%\right)$ are compensated by the inclusion of integral action in the RMPC controller.

As will be seen in the Results section, this seemingly simple control task may become very challenging because of the uncertain input delay.

Constraints on the control input $u_{p}$ were imposed in view of the following physical limitations. Experimental tests 


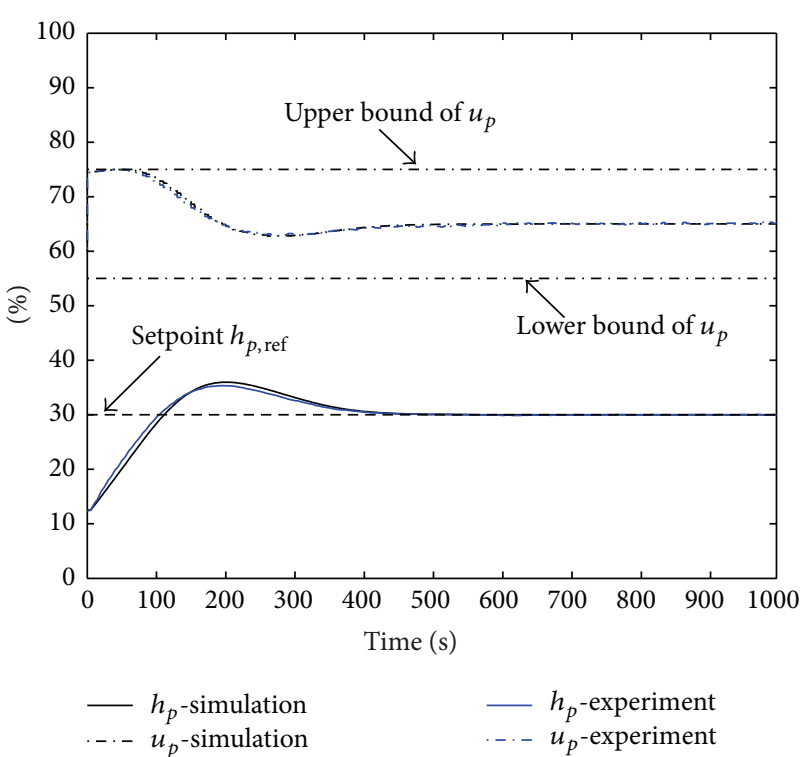

Figure 2: Results for the plant with nominal delay $\left(\tau_{p}=1\right)$, using the nominal predictive controller $(\tau=1)$.

revealed that the pump was unable to elevate water from the reservoir tank $T_{R}$ to the process tank $T_{P}$ if the motor rotation was smaller than $40 \%$. On the other hand, if the rotation was increased beyond $75 \%$, the flow from $T_{R}$ to $T_{P}$ became larger than the input flow to the reservoir tank, which would eventually cause the reservoir to become empty. For these reasons, it is convenient to constrain the control input to the range $40 \% \leq u_{p} \leq 75 \%$. Therefore, in view of (34) with $u_{p, \text { ref }}=65 \%$, the upper and lower bounds on the variable $u$ should be $-25 \%$ and $10 \%$. Since the input constraints must be symmetric in the adopted RMPC approach, the bounds on $u$ were set to $\pm 10 \%$ (corresponding to $55 \% \leq u_{p} \leq 75 \%$ ).

The robust predictive controller (RMPC) was designed by using the state vector (36) augmented with the integrator state $w(k)$. The weight matrices $S$ and $R$ were set to

$$
S=\operatorname{diag}\left[\rho_{h}, \frac{\rho_{u}}{(\bar{\tau}+1)}, \ldots, \frac{\rho_{u}}{(\bar{\tau}+1)}, \rho_{w}\right], \quad R=\frac{\rho_{u}}{(\bar{\tau}+1)},
$$

where diag[.] indicates the elements of a diagonal matrix and $\rho_{h}=1, \rho_{u}=0.1$, and $\rho_{w}=0.01$ are the weights corresponding to the water level $h(k)$, control $u(k)$, and integrator state $w(k)$. A normalization factor $1 /(\bar{\tau}+1)$ is included in (37) to account for the fact that the control appears $(\bar{\tau}+1)$ times in the cost function, once in the control cost itself and $\bar{\tau}$ times in the state cost, since $u(k-1), u(k-2), \ldots, u(k-\bar{\tau})$ are used as state variables.

For comparison, a nominal predictive controller was designed by using the formulation presented in Section 2.3 with $\bar{\tau}=\underline{\tau}=1$. In this case, the polytopic model (1) has a single vertex, which corresponds to the plant without the additional delay. The state vector employed in this formulation is given by

$$
x(k)=[h(k) u(k-1)]^{T}
$$
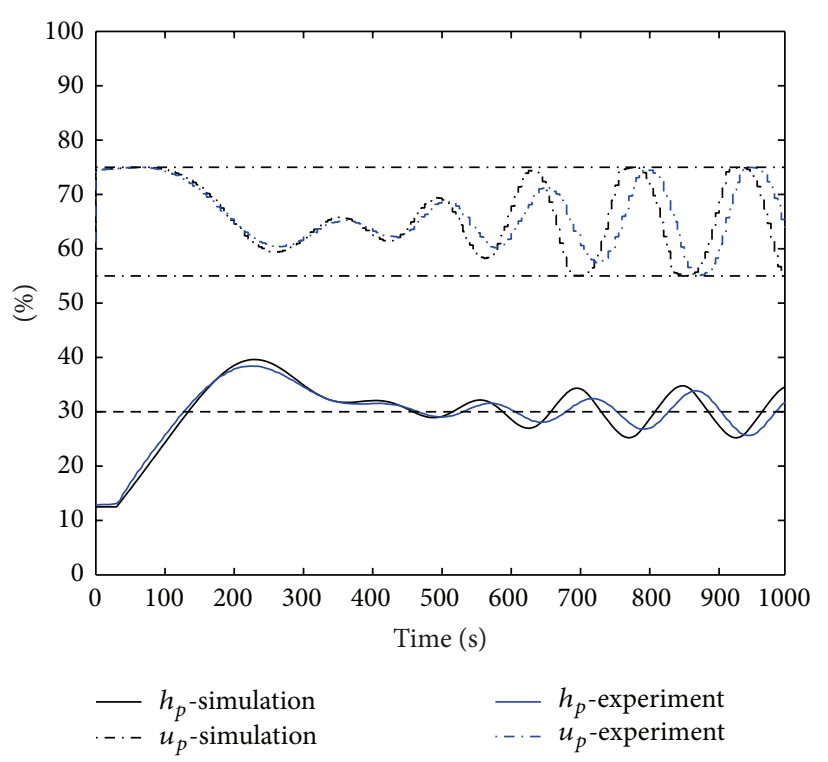

FIGURE 3: Results for the plant with additional delay $\left(\tau_{p}=6\right)$, using the nominal predictive controller $(\tau=1)$.

which is also augmented with the integrator state $w(k)$. The weight matrices $S$ and $R$ were set to

$$
S=\operatorname{diag}\left[\rho_{h}, \frac{\rho_{u}}{2}, \rho_{w}\right], \quad R=\frac{\rho_{u}}{2}
$$

with $\rho_{h}=1, \rho_{u}=0.1$, and $\rho_{w}=0.01$, as in the design of the robust controller.

The following section begins with a comparison between the nominal and robust predictive controllers, in order to illustrate the problems that may arise if the delay uncertainty is not taken into account. Afterwards, the effects of the proposed integrator resetting procedure are discussed. For this purpose, the simplified resetting test (31) was employed, with a cost threshold $\bar{\gamma}=7.5 \times 10^{6}$.

\section{Results and Discussion}

In what follows, the delay considered in the design of the controller will be denoted by $\tau$, whereas the actual delay of the plant will be denoted by $\tau_{p}$. The nominal delay case corresponds to $\tau_{p}=1$ and the case with additional delay introduced in the software environment corresponds to $\tau_{p}=$ 6.

In addition to the experimental outcomes, simulation results are depicted in all graphs to illustrate the validity of the model presented in Section 4.1.

Figure 2 shows the results obtained by using the nominal predictive controller, which considers a delay $\tau=1$ in the plant model. In this case, no additional delay was introduced in the plant and thus $\tau_{p}$ was also equal to 1 . As can be seen, the water level $h_{p}$ converges to the setpoint $h_{p \text {,ref }}$ and the input constraints $\left(55 \% \leq u_{p} \leq 75 \%\right)$ are properly enforced. The simulation and experimental results are in good agreement, which indicates that the adopted model provides an appropriate description of the plant dynamics. 


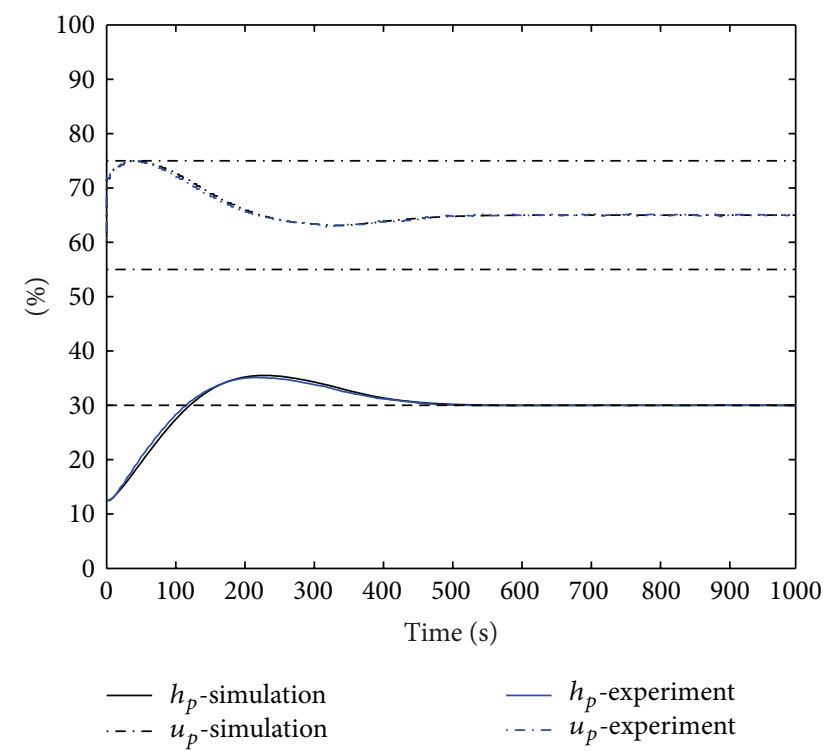

FIGURE 4: Results for the plant with nominal delay ( $\left.\tau_{p}=1\right)$, using the robust predictive controller $(1 \leq \tau \leq 6)$.

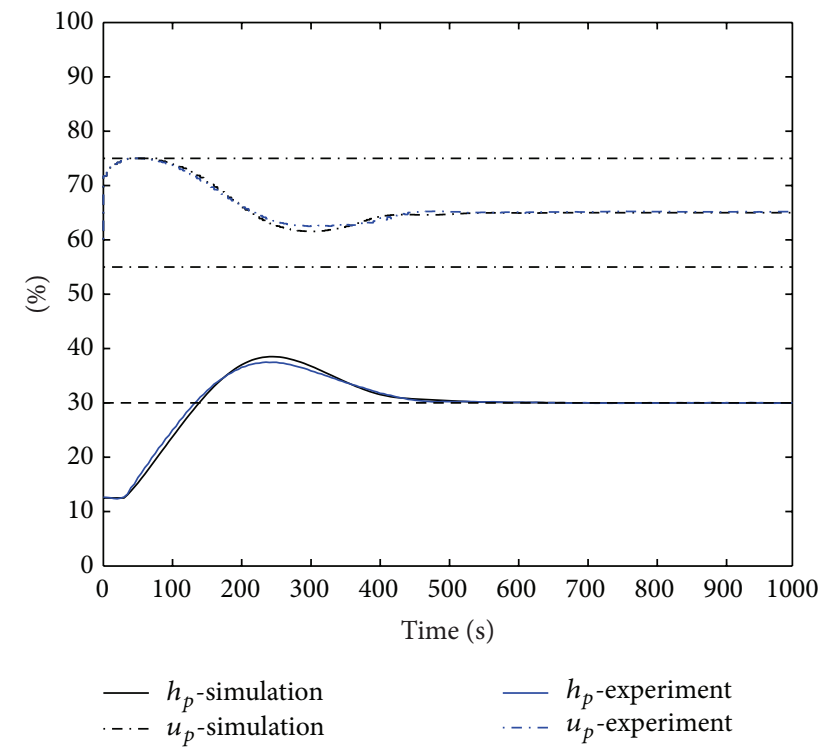

FIGURE 5: Results for the plant with additional delay $\left(\tau_{p}=6\right)$, using the robust predictive controller $(1 \leq \tau \leq 6)$.

Figure 3 shows the case where an additional delay of five sampling periods was introduced in the software environment. Since the plant already has a nominal delay of one sampling period, the overall delay becomes $\tau_{p}=6$. The mismatch between this overall delay and the delay assumed in the design of the nominal predictive controller $(\tau=1)$ results in the loss of closed-loop stability. Indeed, the system response enters a limit cycle rather than converging to the setpoint. The difference between simulation and experimental results can be explained because the model presented in Section 4.1, which was experimentally obtained from small step disturbances around the equilibrium, does not fully

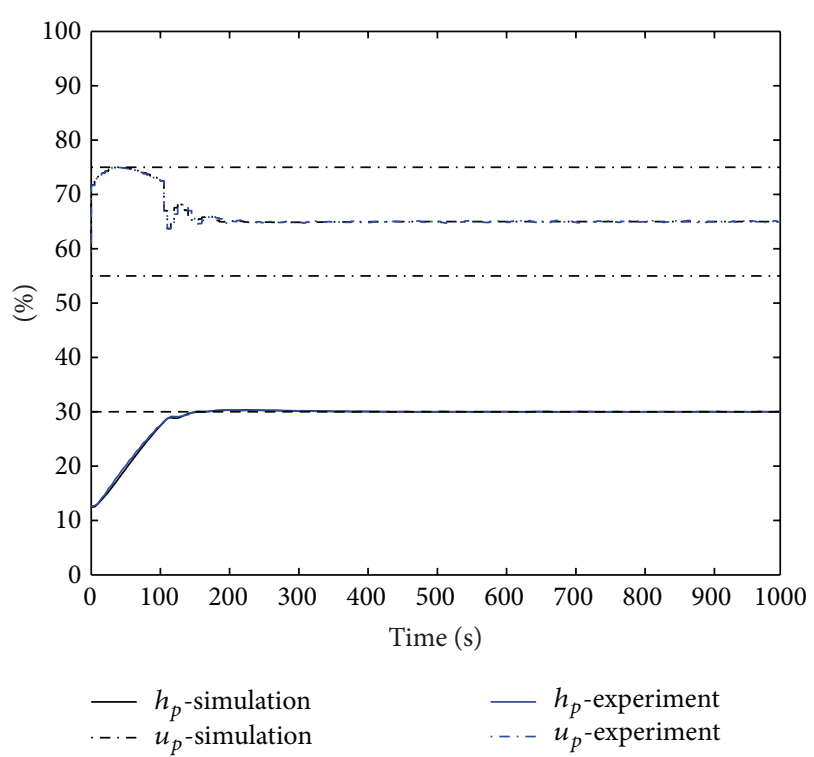

FIGURE 6: Results for the plant with nominal delay ( $\tau_{p}=1$ ), using the robust predictive controller $(1 \leq \tau \leq 6)$ with integrator resetting.

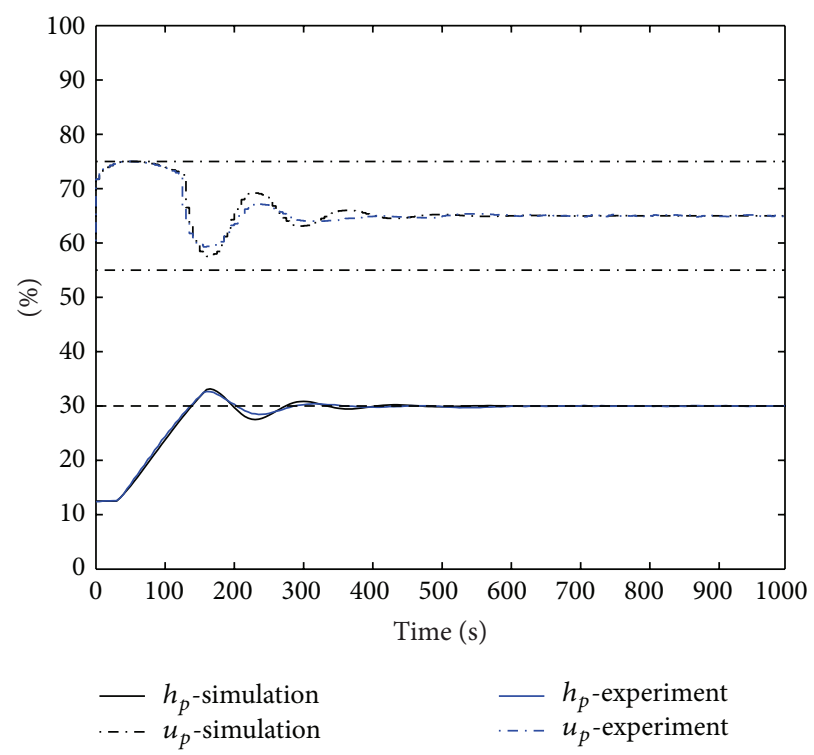

FIGURE 7: Results for the plant with additional delay $\left(\tau_{p}=6\right)$, using the robust predictive controller $(1 \leq \tau \leq 6)$ with integrator resetting.

capture the dynamics involved in the transition to a limit cycle.

As can be seen, even for this simple water level control task, an increase in the time delay can have serious effects on the closed-loop operation. This finding underlines the convenience of using a robust predictive controller (RMPC) to handle possible delays in the range $1 \leq \tau \leq 6$, as described in Section 4.2.

Figures 4 and 5 show the RMPC results obtained in the extreme cases, which correspond to the plant with minimum delay $\left(\tau_{p}=1\right)$ and maximum delay $\left(\tau_{p}=6\right)$, respectively. As can be seen, in both figures the tank level $h_{p}$ converges 
TABLE 1: Experimental values of overshoot $\left(M_{p}\right)$ and settling time $\left(t_{s}\right)$ obtained by using the robust predictive controller with and without integrator resetting.

\begin{tabular}{lccc}
\hline Delay $\tau_{p}$ & Resetting & $M_{p}(\%)$ & $t_{s}(\mathrm{~s})$ \\
\hline 1 & No & 5.1 & 425 \\
6 & No & 7.5 & 440 \\
1 & Yes & 0.3 & 130 \\
6 & Yes & 2.7 & 270 \\
\hline
\end{tabular}

to the setpoint, with satisfaction of the input constraints. Moreover, the transient responses in the two cases are very similar, which corroborates the robustness of the predictive controller. A slightly larger overshoot is observed for the case of maximum delay (Figure 5), which is to be expected since an increase in the time delay tends to deteriorate the closedloop performance.

The transient performance obtained with the RMPC controller can be improved by using the integrator resetting procedure described in Section 3.2. The results are shown in Figures 6 and 7 for the cases of minimum delay $\left(\tau_{p}=1\right)$ and maximum delay $\left(\tau_{p}=6\right)$, respectively. A comparison with Figures 4 and 5 reveals that a substantial reduction was achieved in terms of overshoot and settling time, as summarized in Table 1.

It is worth noting that the overshoot $\left(M_{p}\right)$ indicated in Table 1 corresponds to the difference between the maximum value of $h_{p}$ and the setpoint $h_{p \text {,ref }}=30 \%$. For example, the first entry $\left(M_{p}=5.1 \%\right)$ indicates that $h_{p}$ reached a maximum value of $35.1 \%$. The settling time $t_{s}$ was determined by using a 0.05 criterion. Since the step size from the initial value $h_{p}(0)=$ $12.5 \%$ to the setpoint $h_{p \text {, ref }}=30 \%$ was $17.5 \%$, the settling time $t_{s}$ is the time required for $h_{p}$ to reach and stay within the range $30 \% \pm(0.05 \times 17.5 \%)$, that is, $29.125 \% \leq h_{p} \leq 30.875 \%$.

In short, the results obtained in this case study show that the RMPC formulation is indeed of value to ensure closed-loop stability in the presence of input constraints and uncertain time delays. Moreover, the integrator resetting procedure is convenient to improve the transient response, by reducing both overshoot and settling time in a substantial manner.

\section{Conclusions}

This paper reported experimental results involving the use of an RMPC technique for the control of water level in a pilot plant with actual industrial components. Stability and offsetfree tracking of the setpoint were achieved in the presence of uncertain time delays and control input constraints. Moreover, an integrator resetting procedure based on the concept of regions of guaranteed cost was proposed to improve the closed-loop transient response. The resulting control formulation provided fast and well damped responses, either in the case of nominal delay, or in the case where additional delay was introduced in the plant.

Future research could be concerned with the use of less conservative LMI formulations [14-17] for the deployment of RMPC controllers in experimental settings. Moreover, it would be interesting to carry out studies with other laboratory or field systems that exhibit actual time-delay variations in inputs or state variables. Finally, computational issues may need to be taken into account to allow the application of RMPC to plants with fast dynamics, such as electromechanical systems.

\section{Conflict of Interests}

The authors declare that there is no conflict of interests regarding the publication of this paper.

\section{Acknowledgments}

The authors acknowledge the support of FAPESP (research Grant 2011/17610-0), CAPES (Pró-Engenharias MSc scholarship) and $\mathrm{CNPq}$ (doctoral scholarship and research fellowship).

\section{References}

[1] M. Krstic, "Compensation of infinite-dimensional actuator and sensor dynamics: nonlinear and delay-adaptive systems," IEEE Control Systems Magazine, vol. 30, no. 1, pp. 22-41, 2010.

[2] J. Richard, "Time-delay systems: an overview of some recent advances and open problems," Automatica, vol. 39, no. 10, pp. 1667-1694, 2003.

[3] G. Zong, W. X. Zheng, L. Wu, and Y. Yi, "New developments in time-delay systems and its applications in engineering," Mathematical Problems in Engineering, vol. 2013, Article ID 198245, 2 pages, 2013.

[4] E. Fridman and U. Shaked, "Delay-dependent $H_{\infty}$ control of uncertain discrete delay systems," European Journal of Control, vol. 11, no. 1, pp. 29-37, 2005.

[5] E. Boukas, "Discrete-time systems with time-varying time delay: stability and stabilizability," Mathematical Problems in Engineering, vol. 2006, Article ID 42489, 10 pages, 2006.

[6] X. G. Liu, R. R. Martin, M. Wu, and M. L. Tang, "Delaydependent robust stabilisation of discrete-time systems with time-varying delay," IEE Proceedings. Control Theory \& Applications, vol. 153, no. 6, pp. 689-702, 2006.

[7] J. M. Maciejowski, Predictive Control with Constraints, Prentice Hall, Harlow, UK, 2002.

[8] X. Liu, H. Nong, K. Xi, and X. Yao, "Robust distributed model predictive load frequency control of interconnected power system," Mathematical Problems in Engineering, vol. 2013, Article ID 468168, 10 pages, 2013.

[9] P. J. Campo and M. Morari, "Robust model predictive control," in Proceedings of the American Control Conference, pp. 10211026, Minneapolis, Minn, USA, 1987.

[10] J. C. Allwright and G. C. Papavasiliou, "On linear programming and robust model-predictive control using impulse-responses," Systems \& Control Letters, vol. 18, no. 2, pp. 159-164, 1992.

[11] Z. Q. Zheng and M. Morari, "Robust stability of constrained model predictive control," in Proceedings of the American Control Conference, pp. 379-383, San Francisco, Calif, USA, June 1993.

[12] M. V. Kothare and V. Balakrishnan, "Robust constrained model predictive control using linear matrix inequalities," Automatica, vol. 32, no. 10, pp. 1361-1379, 1996. 
[13] S. Boyd, L. El Ghaoui, E. Feron, and V. Balakrishnan, Linear Matrix Inequalities in System and Control Theory, SIAM, Philadelphia, Pa, USA, 1994.

[14] F. A. Cuzzola, J. C. Geromel, and M. Morari, "An improved approach for constrained robust model predictive control," Automatica, vol. 38, no. 8, pp. 1183-1189, 2002.

[15] W. J. Mao, "Robust stabilization of uncertain time-varying discrete systems and comments on "an improved approach for constrained robust model predictive control'"' Automatica, vol. 39, no. 6, pp. 1109-1112, 2003.

[16] N. Wada, K. Saito, and M. Saeki, "Model predictive control for linear parameter varying systems using parameter dependent Lyapunov function," IEEE Transactions on Circuits and Systems II: Express Briefs, vol. 53, no. 12, pp. 1446-1450, 2006.

[17] S. M. Lee and J. H. Park, "Output feedback model predictive control for LPV systems using parameter-dependent Lyapunov function," Applied Mathematics and Computation, vol. 190, no. 1, pp. 671-676, 2007.

[18] Z. Wan and M. V. Kothare, "Robust output feedback model predictive control using off-line linear matrix inequalities," Journal of Process Control, vol. 12, no. 7, pp. 763-774, 2002.

[19] Z. Wan and M. V. Kothare, "A framework for design of scheduled output feedback model predictive control," Journal of Process Control, vol. 18, no. 3-4, pp. 391-398, 2008.

[20] B. Ding, Y. Xi, M. T. Cychowski, and T. O’Mahony, "A synthesis approach for output feedback robust constrained model predictive control," Automatica, vol. 44, no. 1, pp. 258-264, 2008.

[21] Z. Wan and M. V. Kothare, "Efficient scheduled stabilizing model predictive control for constrained nonlinear systems," International Journal of Robust and Nonlinear Control, vol. 13, no. 3-4, pp. 331-346, 2003.

[22] M. S. M. Cavalca, R. K. Galvão, and T. Yoneyama, "Robust model predictive control using linear matrix inequalities for the treatment of asymmetric output constraints," Journal of Control Science and Engineering, vol. 2012, Article ID 485784, 7 pages, 2012.

[23] B. Ding and B. Huang, "Constrained robust model predictive control for time-delay systems with polytopic description," International Journal of Control, vol. 80, no. 4, pp. 509-522, 2007.

[24] B. Ding, "Robust model predictive control for multiple time delay systems with polytopic uncertainty description," International Journal of Control, vol. 83, no. 9, pp. 1844-1857, 2010.

[25] B. Ding, L. Xie, and W. Cai, "Robust MPC for polytopic uncertain systems with time-varying delays," International Journal of Control, vol. 81, no. 8, pp. 1239-1252, 2008.

[26] G. Huang and S. Wang, "Use of uncertainty polytope to describe constraint processes with uncertain time-delay for robust model predictive control applications," ISA Transactions, vol. 48, no. 4, pp. 503-511, 2009.

[27] R. Waschburger and R. K. H. Galvao, "Influence of the delay in a robust predictive controller employing linear matrix inequalities," in Proceedings of the 10th Simposio Brasileiro de Automacao Inteligente (SBAI '11), pp. 1202-1207, São João del Rey, Brazil, 2011 (Portuguese).

[28] F. Q. Rossi, R. Waschburger, and R. K. H. Galvao, "Regions of guaranteed cost for LMI-based robust model predictive controllers for systems with uncertain input delay," in Proceedings of the Conference on Control and Fault-Tolerant Systems (SysTol '13), pp. 584-589, Nice, France, 2013.

[29] M. S. Ramli, "Improved coupled tank liquid levels system based on swarp adaptative tuning of hybrid proportional-integral neural network controller," American Journal of Engineering and Applied Sciences, vol. 2, no. 4, pp. 669-675, 2009.

[30] E. P. Gatzke, E. S. Meadows, C. Wang, and F. J. Doyle III, "Model based control of a four-tank system," Computers and Chemical Engineering, vol. 24, no. 2-7, pp. 1503-1509, 2000.

[31] R. Dormido, H. Vargas, N. Duro et al., "Development of a webbased control laboratory for automation technicians: the threetank system," IEEE Transactions on Education, vol. 51, no. 1, pp. 35-44, 2008.

[32] J. S. B. Lopes, O. G. Filho, F. M. U. Araujo, A. L. O. Cavalcanti, and A. L. Maitelli, "An LMI robust predictive control approach applied in a coupled tanks systems," in Proceedings of the 37th Annual Conference on IEEE Industrial Electronics Society (IECON'11), pp. 480-485, Melbourne, Australia, November 2011.

[33] M. S. M. Cavalca, R. K. H. Galvão, and T. Yoneyama, "Integrator resetting with guaranteed feasibility for an LMI-based robust model predictive control approach," in Proceedings of the 18th Mediterranean Conference on Control and Automation (MED '10), pp. 634-639, Marrakech, Morocco, June 2010.

[34] F. Q. Rossi, R. Waschburger, and R. K. H. Galvao, "Determination of the domain of attraction and regions of guaranteed cost for robust model predictive controllers based on linear matrix inequalities," in Proceedings of the UKACC International Conference on Control (CONTROL '12), pp. 982-987, Cardiff, UK, September 2012.

[35] R. Waschburger, Robust predictive controllers for systems with uncertain transport delay, [Doctoral thesis], Instituto Tecnológico de Aeronáutica, São José dos Campos, Brazil, 2013, (Portuguese).

[36] G. F. Franklin, J. D. Powell, and M. L. Workman, Digital Control of Dynamic Systems, Addison-Wesley, Menlo Park, Calif, USA, 3rd edition, 1998.

[37] F. Q. Rossi, Level control of an industrial pilot plant employing predictive controllers with robustness to uncertain input delay [M.S. thesis], Instituto Tecnologico de Aeronautica, São José dos Campos, Brazil, 2014, (Portuguese).

[38] Y. Wang, H. R. Karimi, and Z. Xiang, " $H_{\infty}$ Control for Networked Control Systems with Time Delays and Packet Dropouts," Mathematical Problems in Engineering, vol. 2013, Article ID 635941, 10 pages, 2013. 


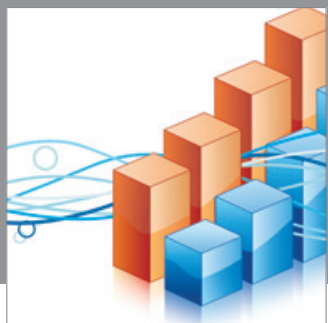

Advances in

Operations Research

mansans

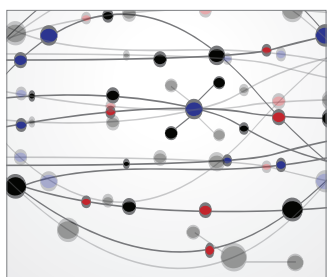

The Scientific World Journal
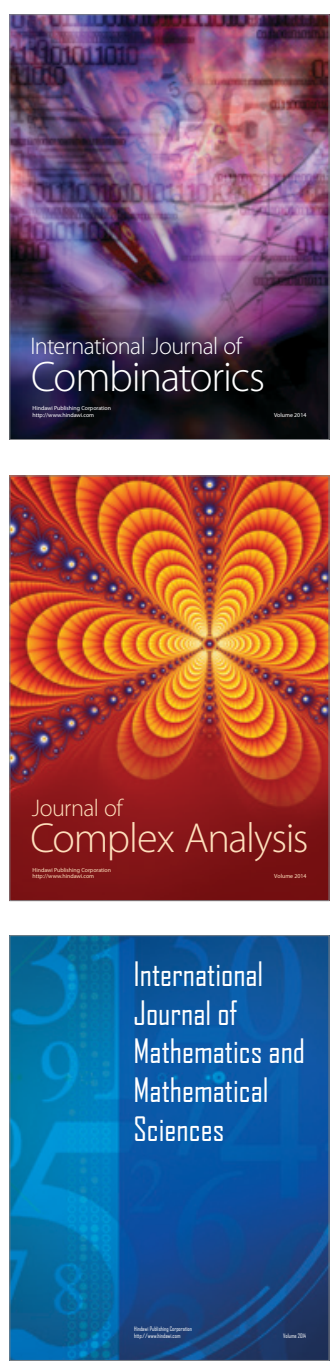
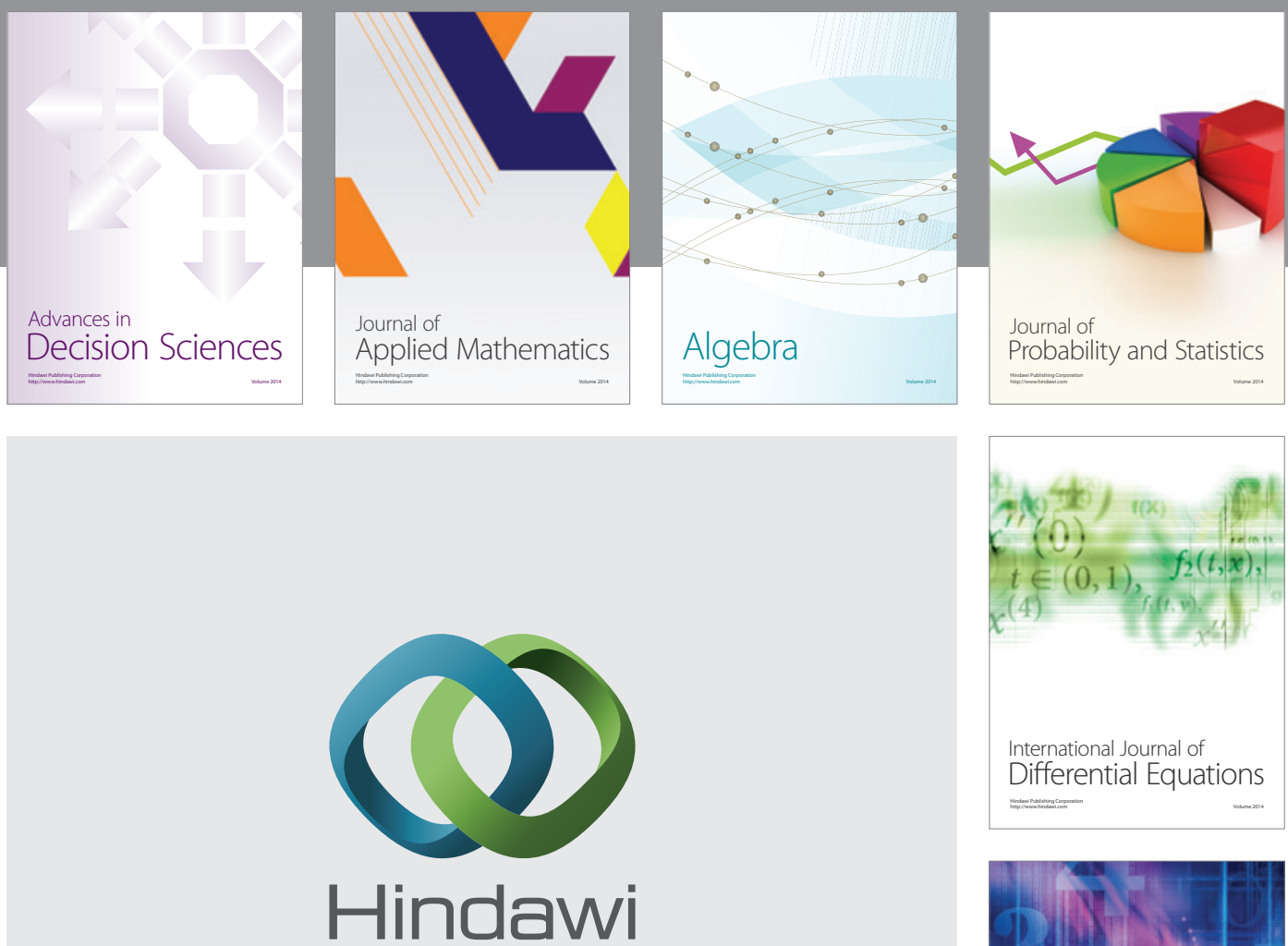

Submit your manuscripts at http://www.hindawi.com
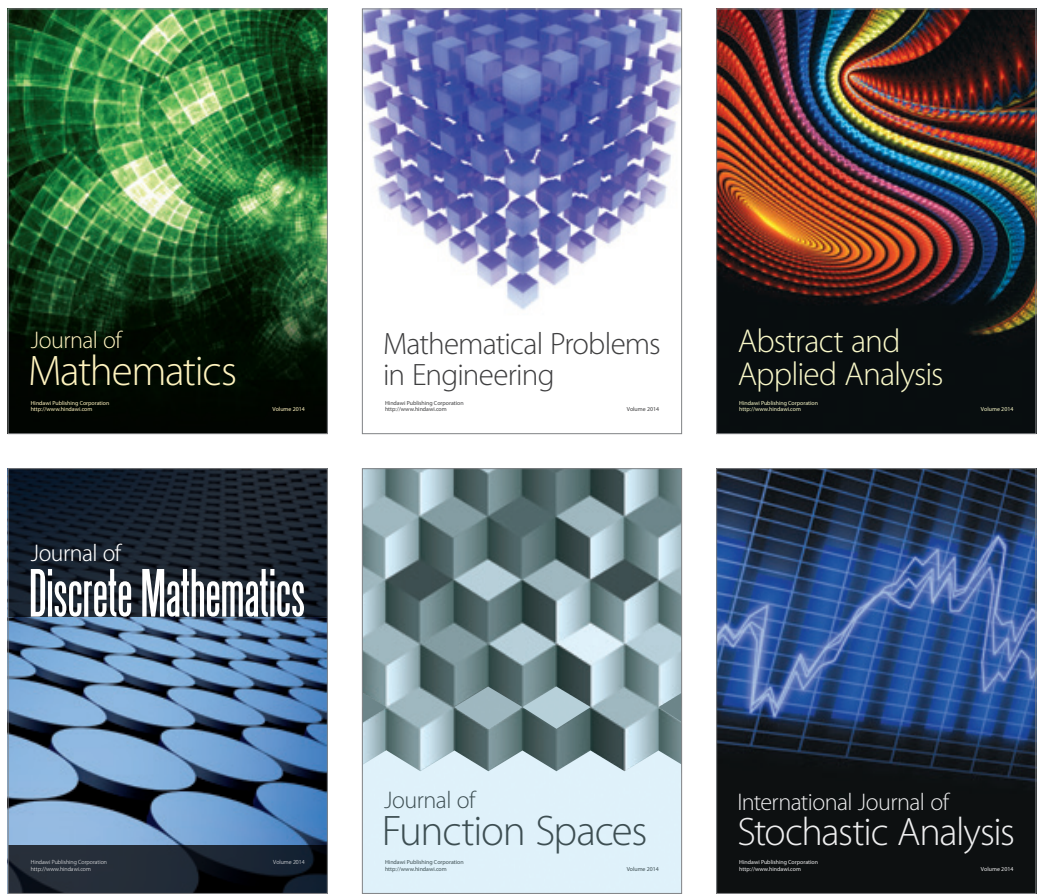

Journal of

Function Spaces

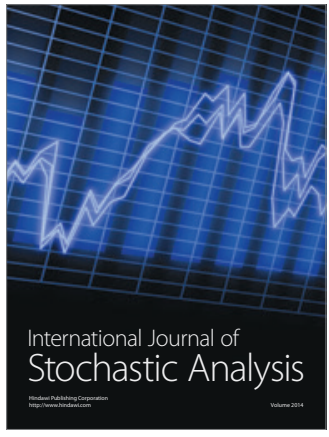

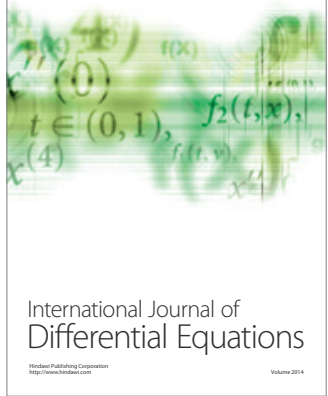
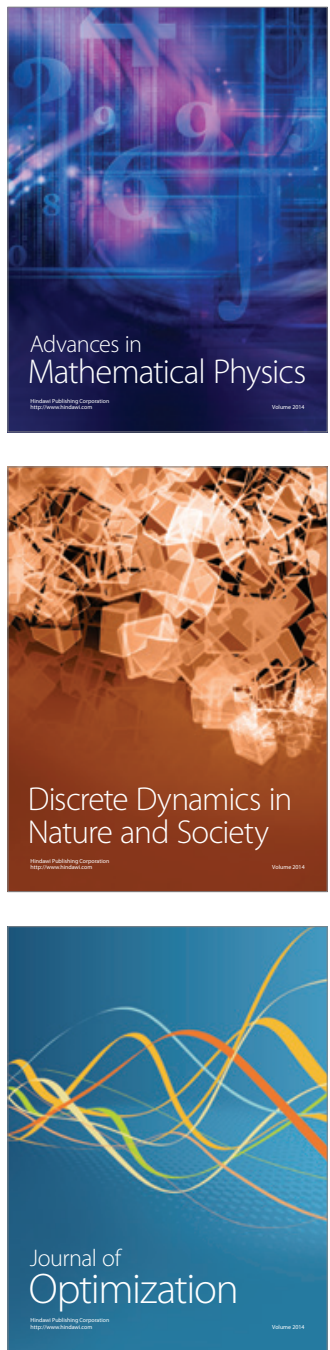